\title{
Exploring the Benefits of a Collaborative Inquiry Team in Education (CITE) Initiative to Develop a Research Community and Enhance Student Engagement
}

\author{
Maria Cantalini-Williams
}

Nipissing University

Debra Curtis

Waterloo Catholic District School Board

Kimberley Eden-DeGasperis

Waterloo Catholic District School Board

Lauren Esposto

Waterloo Catholic District School Board

Jenny Guibert

Nipissing University

Heather Papp

Waterloo Catholic District School Board

Carlos Roque

Waterloo Catholic District School Board

\begin{abstract}
This study examined a collaborative inquiry process, facilitated by university faculty in an elementary school, intended to develop a research community, foster knowledge mobilization, and enhance student engagement. The Collaborative Inquiry Team in Education (CITE) initiative consisted of five school-based sessions that included videos, discussions, and the completion of a research action plan. Data collection and analysis involved sessions' transcripts, feedback from participants, documents such as brainstorming charts, and student artifacts. Findings indicate that the collaborative inquiry process with enablers of time, flexibility, and support from university faculty increased educators' research acumen and student engagement in classrooms. The CITE initiative is an effective example of applied education research and knowledge mobilization with the inclusion of faculty and technological support, innovative resources, and the co-construction of new understandings.
\end{abstract}

Keywords: collaborative inquiry, school-university partnerships, educational research, knowledge mobilization, student engagement, teacher engagement 
Maria Cantalini-Williams is a professor and former board consultant. Interests include experiential learning, early childhood education, and applied research.

mariac@ nipissingu.ca

Debra Curtis is an elementary school principal. Interests include goal-setting for student achievement, staff development and, collaborative learning teams.

debra.curtis@wcdsb.ca

Kimberley Eden-DeGasperis is a literacy/numeracy teacher. Interests include effective teaching strategies and building of content knowledge across the curriculum.

kimberley.degasperis@wcdsb.ca

Lauren Esposto is an elementary school teacher. Interests include Christian Meditation and effective teaching practices for student learning.

lauren.esposto@wcdsb.ca

Jenny Guibert is an early learning instructor. Interests include pedagogical practices, teacher development, and environmental education.

jennyg@nipissingu.ca

Heather Papp is an intermediate division teacher. Interests include support of mental health, equity and inclusion issues, and differentiated instruction for student learning.

heather.papp@wcdsb.ca

Carlos Roque is an elementary school teacher. Interests include technology, equity, collaborative learning, and reflective practice.

carlos.roque@wcdsb.ca 


\section{Introduction}

The Ministry of Education (MOE) in Ontario has undertaken some initiatives to support the development of a community of educational researchers among stakeholder groups who work with students in teaching and learning organizations. For more than a decade, the MOE has recognized the importance of research for educators and has instituted a Research and Evaluation Strategy, fostering collaboration between partners in education. The annual Ontario Education Research Symposium (OERS), held since 2005, and the Knowledge Network for Applied Education Research (KNAER) grants which began in 2009 are evidence of the government's commitment to educational research and knowledge mobilization (KMb). In the MOE London region, the School Board-University Research Exchange (SURE) Network was formed as a partnership among school board personnel and university faculty. SURE members have been actively engaged in a range of initiatives to encourage $\mathrm{KMb}$ and collaborative educational research among school board and university partners. These initiatives include joint conferences, publications, and the development of media productions such as the SURE Network's (2014) Research to Practice video series sponsored by a Social Sciences and Humanities Research Council (SSHRC) Connection grant. The Ontario Ministry of Education (OME) has also provided the Capacity Building Series and the Ontario Education Research Panel (OERP) publications describing the collaborative inquiry process (OME, 2016). These documents, developed over many years, reiterate the need for building mutual interest, respect, and trust among educators and researchers, as well as the commitment to a flexible research design in education-related collaborative inquiry initiatives.

This paper describes a study examining an initiative implemented to actively engage educators of a school in the Waterloo Catholic District School Board (WCDSB) in professional development, research, and $\mathrm{KMb}$ activities through the formation of a Collaborative Inquiry Team in Education (CITE). The CITE initiative was led by two university faculty members who had previously worked for the WCDSB and one had served as a co-chair of the School BoardUniversity Exchange (SURE) steering committee for two years. CITE was comprised of a series of collaborative inquiry sessions to promote education research and to address challenges outlined in the school's improvement plan. The CITE initiative utilized a variety of resources including the video series developed by SURE (see http://www.surenetwork.ca/). These videos were produced by SURE as knowledge mobilization tools, and in this study, were hypothesized to foster research acumen among the CITE school participants and to stimulate the collaborative inquiry process. The CITE participants became interested in reflecting on their practices and pedagogies to promote such areas of student engagement as resilience, persistence, independence, and self-efficacy. By examining their school's third and sixth grade provincial assessment data, the CITE school participants determined that increasing student engagement was a "challenge of practice," requiring increased attention and purposeful strategies. The overall collaborative inquiry focus became improving student engagement through effective instructional strategies.

The design of the collaborative inquiry was tailored and developed to address the emerging inquiry questions of participating teachers and the learning needs identified through their school's improvement plan. KMb was fostered through the partnership with the university faculty and the utilization of the SURE video resources. The initiative was based on the applied education research community theoretical framework (Martinovic, Wiebe, Ratković, WillardHolt, Spencer, \& Cantalini-Williams, 2012). The teacher as researcher concept, with faculty of

Brock Education Journal, 25 (1), Fall 2015 
education support, was explored and actualized through this framework. The initial goal was to involve teachers in a professional development and research-based endeavour with the authentic purpose of facilitating $\mathrm{KMb}$ and enhancing student engagement and learning.

\section{Literature Review}

Teachers as researchers have a unique opportunity to bridge the gap between theory and practice in ways that benefit teacher knowledge, contribute to life-long learning, and empower teachers to question and improve current educational practices (Kyei-Blankson, 2014). Collaborative inquiry models allow teachers to become a part of a community of researchers, addressing educational issues and building on the individually-based action research model (Hannay, Wideman, \& Seller, 2010). Teachers have been found to be ideal researchers as they are insightful and knowledgeable in regards to current teaching practices and student needs (Blakemore, 2012). Such research with teacher involvement is suggested to be a valid and valuable method for gaining a deeper understanding of educational issues in today's classrooms (Hine \& Lavery, 2014).

Collaborative inquiry includes teachers as crucial members of an information culture in education (Williams \& Coles, 2007). Initiatives to support collaborative inquiry teams within the educational setting help establish knowledge and create schools that lead to improvements in current teaching practices (McIntyre, 2005). Partnerships have proven to be especially successful when universities, schools, and teachers work in collaboration to conduct meaningful research about current educational issues (Gore \& Gitlin, 2004; Kyei-Blankson, 2014). In addition to collaboration, such elements as communication, commitment, and continuity of the research team are also important to the success of such research programs (Ponte, Beijard, \& Ax, 2004). Kuntz, Presnall, Priola, Tilford, and Ward (2013) found that classroom research fosters a sense of agency and ability within teacher researchers. Teacher researchers are often empowered by their new dual-role (Kane \& Chimwayange, 2014) and able to decrease the divide between knowledge construction and knowledge mobilization.

Collaborative inquiry teams are a successful method of gaining and mobilising important information about educational issues (Blakemore, 2012; Gabriel, Doiron, Baldacchino, McKenna, \& O'Keefe, 2012). Gabriel et al. (2012) conducted a qualitative research study and examined several educational issues including mixed-age groupings, initiative approach learning, and integration of literacy in other curriculum areas. Their findings indicated that engaging and supporting teachers as researchers were effective means of improving educational practices. Additionally, the authors found that the teachers were interested in continuing their research as the research project provided a rich learning opportunity that would not have been otherwise available to them. These findings indicate the value of collaborative inquiry and a research culture that supports teachers as researchers in addressing important issues in their field.

In a seminal work on $\mathrm{KMb}$, Cooper (2011) found that $\mathrm{KMb}$ is an effective strategy for the exchange of knowledge between academic research and professional practice. Recently, Cooper (2014) examined KMb efforts and noted that only a small percentage of researchers used online strategies for dissemination and discussion of their information. This utilization of technology for $\mathrm{KMb}$, or more specifically for collaborative research and inquiry, is a new

phenomenon that merits further study and has been included in the CITE initiative. Collaborative inquiry models where teachers experience the dual role of teacher and researcher within a 
collegial culture of inquiry may help bridge the gap between theory and practice in meaningful and authentic ways.

The theoretical framework for the exploration of the CITE initiative is based on the findings of a large-scale study exploring teachers' perceptions of education research (Martinovic et al., 2012). In this Ontario-based study, education research was viewed as demonstrating evidence of success when collaboration is utilized and teachers feel empowered to engage in first-hand research (Martinovic et al., 2012). The study participants (i.e., teachers across various school boards and elementary/secondary panels) identified benefits, enablers, and challenges for those educators who are involved or interested in research.

Based on the applied education research community framework (Martinovic et al., 2012), the elements and enablers described as necessary for effective collaborative research initiatives were integrated into the CITE initiative. The CITE initiative is an example of the Applied Education Research Community (Martinovic et al., 2012) because it is collaborative inquiry characterized by ethical review, research questions and methods, data collection and analysis, dissemination of findings, and potential improvement of teaching and learning. Various challenges as outlined in the described framework were also identified and addressed in the processes of CITE. Additionally, the concept of KMb described by Cooper (2011) provided a lens for understanding the co-creation and exchange of understandings across academic and practitioner communities.

To engage teachers in research processes and $\mathrm{KMb}$, especially through a collaborative inquiry team approach, university faculty are hypothesized to be instrumental in this process (Martinovic et al., 2012). In Ontario, there is a call for academics and practitioners to work together by sharing ideas and addressing common educational issues, yet there is little research on the role of university faculty in guiding collaborative inquiry teams. The CITE initiative was created to address this research area through faculty facilitation of a school-based inquiry. The use of $\mathrm{KMb}$ strategies, collegial and technological support, and a structured format for knowledge exchange were expected to increase positive perceptions of the collaborative inquiry process and to foster teacher and student engagement.

\section{Methodology}

The methodology of this research project is similar to that of the collaborative self-study conducted by Grierson, et al. (2012). Grierson et al. (2012) collected data from a range of sources such as participant questionnaires, transcripts of group discussions, and related artifacts. The authors found that participating faculty who documented their discussions and perceptions in relation to teacher education over a series of sessions, not only learned about their respective roles and tensions as teacher educators, but also formed a professional learning research community. Kitchen, Ciuffetelli Parker, and Gallagher (2008) also documented the benefits of collaborative self-study as a form of faculty development, highlighting how it promoted engagement in authentic conversations. Latta and Buck (2007) found that there are potential risks and opportunities in a collaborative self-study, yet it is a valuable vehicle for professional development. Reflection was a major component of the CITE initiative, as Leigh (2016) reported, that reflexivity implies individuals take time to reflect on and analyse their actions and awareness of themselves, and then use those insights to improve their practice.

The present study is a form of collaborative self-study designed to address the following research questions: 
1. How does the collaborative inquiry process facilitate participants' awareness of and interest in the research process?

2. How does the collaborative inquiry process create a community of educational researchers?

3. How does the collaborative inquiry process contribute to increased competencies and changes in pedagogical practices?

4. How did the CITE initiative enhance student learning and skills?

5. What are the enablers and challenges to developing collaborative inquiry teams in education?

This study included the implementation of a series of five collaborative inquiry sessions over a 5-month period with a total of eleven participants including the two university faculty facilitators. The data was collected by a university student research assistant throughout the series of collaborative inquiry sessions. The sessions included such agenda items as viewing videos, engaging in team activities, discussing instructional practices, and reflecting on evidence of student learning.

\section{Context and Participants}

The setting is a school within the Waterloo Catholic District School Board. It is a mid-sized school with a diverse socioeconomic population and below average scores from Education Quality and Accountability Office (EQAO) assessments. There were nine school board educators who volunteered as members of the collaborative inquiry group and they all agreed to participate in the present study. Six of the participants were classroom teachers and represented a crosssection of grade levels, ranging from Grades 1 to 7 . Other participants included the Board Student Work Study (SWS) teacher who assisted in setting up the CITE initiative, the Literacy/Numeracy resource teacher and the school principal who all attended each session as active participants. The two faculty facilitators of the CITE initiative planned the sessions and led the discussions. They participated fully in the collaborative inquiry, but their comments are not included in the data and findings which include only the perceptions of nine school and board personnel.

The classroom teachers received release time from a board budget to participate in the CITE initiative. Some of the teachers had previously worked with the SWS teacher, who invited them to participate in the CITE initiative; others responded to an email invitation sent to all teachers by the principal. The collaborative inquiry team participated willingly in this professional development and research project to increase their understandings of educational research and to enhance student learning. The Nipissing University Research Ethics Board and WCDSB provided research ethics clearance for the study.

\section{Data Sources}

A qualitative approach to data collection and analysis was used. The three main sources of data were transcripts of discussions, school board participants' feedback forms, and artifacts created during session activities. A discussion portion of each session was audiotaped to gather participants' perceptions of the benefits and challenges encountered in the research process and to identify new learnings related to pedagogy and practice. These discussions usually took place 
after watching SURE videos, developing goals/theories of action, and reviewing pedagogical

documentation. Feedback forms were completed anonymously by each participant at the end of each session to allow for reflection and to document examples of learning, and submitted to a research assistant. Various documents such as discussion charts and action plans were created at every session. These artifacts were collected to provide evidence of participant learning.

\section{Data Collection}

At each CITE meeting, all nine school board participants and two University faculty members were present. The meetings were held for approximately three hours in a school seminar room, with Internet access and group table arrangements. The five sessions included purposeful scaffolding of learning and exploration. Each session began with a review of the agenda and ended with the completion of feedback forms by the participants.

The first session was of an introductory nature to familiarize participants with the goals of the CITE initiative and to identify teachers' initial perceptions towards research. The discussion following the viewing of a SURE video (Thinking Like a Researcher) allowed some unpacking of concepts and terms related to teacher research. This video also was intended to teach participants about SURE's KMb efforts. Various documents developed by the Ministry of Education such as the Capacity Building and Research into Practice series were shared with participants and provided a basis for a common understanding. The school principal, as requested by the facilitators, described the main challenge of practice as outlined by the School Improvement Team after a review of the 2014 school provincial assessment data. The CITE participants determined that student persistence in task completion was central to improving student engagement and learning across grades and would become a focus of the collaborative inquiry sessions.

In Session 2, a review of the first session activities served as a reminder of CITE goals and the SURE video on Collaborative Inquiry Models provided many examples of collaborative research conducted in local school boards. In this session, participants became familiar with a template termed a Research Action Plan as introduced by a corresponding video describing the cycle of inquiry. Participants were asked to begin to determine their area of focus within the overall CITE school-wide inquiry question: How can we foster an environment that is conducive to productive engagement for learning?

In Session 3, the participants worked in small groups or pairs to complete their Research Action Plan with an outline of their goals, strategies, resources, data sources, and potential evidence of learning. Teachers refined their classroom focus areas into educational goals such as improving student perseverance to work through challenging problems and demonstrating increased levels of accountability and independence in the learning process. The resource teachers and the principal assisted the classroom teachers to brainstorm possible strategies to address these goals. Teachers became more familiar with the term pedagogical documentation and use of data by viewing a related MOE video on pedagogical documentation and the SURE Video: Research to Practice: Forms and Possibilities. Between Sessions 3 and 4, the classroom teachers implemented instructional strategies related to their area of inquiry such as the use of checklists, rubrics, and focused activities.

In Session 4, the participants began to explore the potential of using a school-based learning management system operated by the Desire 2 Learn (D2L) company, and utilised by WCDSB, to post the CITE materials online and to encourage discussion among participants. One 
teacher was particularly helpful and invited all participants to this site. At this session, OME documents What Works? Research into Practice related to resilience, poverty, and KMb were distributed for discussion. The fourth session was also used to review the project progress and to prepare for a workshop that this group had collectively agreed to facilitate at a SURE conference in London, Ontario on April 30, 2015. Some time was spent on determining content and roles for that workshop facilitation.

The fifth and final session included a sharing of CITE participants' learnings and the classroom teachers showed their pedagogical documentation, such as student work samples, as evidence of students' progress and persistence in learning activities. Participants expressed their perceptions of using D2L for the initiative and discussed expanding this process to include other teachers in their school, and possibly extending the initiative to a nearby partner school.

\section{Data Analysis}

The session transcripts, feedback forms, and session artifacts were analyzed using inductive thematic analysis techniques (Charmaz, 2006; Guest, Namey, \& Mitchell, 2013). This approach involved simultaneous data collection and analysis, coding, constant comparison of codes, identification of emergent themes, memo-writing about category and theme development, and iterative analysis (Charmaz, 2006). Strategies for analysis from Miles and Huberman (1994) were also used in the analysis stage including display of findings, and drawing and verifying conclusions.

The two university researchers and a research assistant examined the transcripts, the feedback forms, and the documents to categorize the information gained from the participants and their discussions. Emergent themes were developed into a model with interrelating categories to inform a theory that further explores the process of collaborative inquiry and supports the proposed framework of applied educational research (Creswell, 2009). The intent of the data analysis was to identify the participants' perceptions of collaborative inquiry in education. To ensure the robustness and rigour of the analysis process, the research team included two university researchers and a research assistant who collected and co-analyzed data, used data triangulation, and offered participants the opportunity to member check session transcripts and main themes.

\section{Findings}

To determine the findings of the present study, the data collected was analyzed according to the five research questions. The participant feedback, available in both oral and written form, was analyzed, along with an analysis of documents such as participants' Research Action Plans and artifacts of student work.

\section{How does the collaborative inquiry process facilitate participants' awareness and interest in the research process?}

The participants felt strongly that the CITE process was extremely beneficial to increase their awareness of the MOE position on educational research and collaborative inquiry. Most participants were not aware of Ministry direction, yet had a moderate comfort level with research protocols and processes before the CITE initiative. None of the participants, except one, were

Brock Education Journal, 25 (1), Fall 2015 
aware of the SURE network, the related website, events, and resources. When asked how confident they were with determining a research topic and collecting evidence, most participants reported that they were moderately comfortable with these steps in the research process. Fewer participants had familiarity with developing research questions and writing a summary report or an article on the findings. Participants were open minded about the CITE initiative and embraced it even though the purpose of the initiative was initially unclear to them. One participant said in the first session: "I found today very useful and am looking forward to embarking on this research initiative," and another reported: "My mind was thinking in a way that engages me." .After involvement in several sessions, one participant reinforced the value of the collaborative inquiry process as a means for reflection and action research stating the following: "It was very helpful to go through each step in the research process and apply it to our area of focus." Another participant noted that the SURE video resources were helpful: "The SURE videos provided real examples of collaborative research. They showed us that research can be easily conducted within a classroom setting."

The classroom teachers appreciated the collegial atmosphere for learning and the opportunity to work together with a common research focus. One teacher noted: "I enjoyed seeing it in action with other colleagues." The teachers learned about the research process as they experienced it as researchers and participants. One teacher said quite emphatically: "I need to have a connection to the research. It needs to be related to my teaching; otherwise, I am not interested." Many commented on the continuous need to revise and reflect on their thinking throughout the research process. A participant in the study said: "I appreciate the ability to gather results/change techniques and tweak strategies," and another noted: "We are recognizing that we need to take the time to really dissect the question and focus before we take action."

The data from the feedback forms and discussions indicated that participants embraced the research experience fully through the collaborative inquiry process. They used terminology related to research such as "research questions," "gather results", and "focus strategies." The participants were very excited to learn that other teachers had also been involved in collaborative inquiry processes with university facilitators as had been demonstrated in the SURE videos. They were especially looking forward to the opportunity to share their research at the SURE conference. Some participants were also very interested in co-authoring the present article.

Attending the SURE conference was an opportunity to reflect on new understandings and develop an interactive workshop. Six teachers were able to mobilise knowledge created through CITE by presenting their preliminary findings to educators and peers from across the province. In the summary session after the SURE conference, the presenters reported that people in the session appreciated the validity of the front-line teacher perspective. One teacher stated that "participants in our session became most interested when we talked about our data and when we mentioned the use of D2L as a research site." Teachers' involvement in both the CITE sessions and in the presentation at the SURE conference increased teachers' awareness and interest in research and $\mathrm{KMb}$ processes.

\section{How does the collaborative inquiry process create a community of educational researchers?}

The CITE initiative provided teachers with an opportunity to work together in a researchfocussed environment, with faculty facilitators and administrative support while remaining on their school site. This was a new experience for many participants allowing them to increase 
awareness of their colleagues' interests and educational issues. The participants clearly articulated the new realization that others had the same challenges and questions as they had in their classrooms. One teacher noted:

I really like the time we were able to show what our concerns were as [we] were trying to gather questions for potential research. Hearing that other teachers are experiencing the same things I am feeling assures me I am on the right track.

Another teacher commented: "Sometimes my mind wonders at 100mph while I am here. But I love how I am thinking about my practice." The participants were particularly appreciative of the time and space that allowed them to discuss, share, and reflect on ideas as indicated by one participant: "It's always useful working with colleagues as it allows us to share ideas about students we are familiar with, and possibly will work with in future years." The collaboration and support, evident in the group, provided a safe setting for generating potential solutions to school-wide issues: "Being able to discuss strategies and next steps for our students in a collaborative setting is helpful." Participants were thankful for having the educational and human resources, such as faculty, the SWS teacher, and the principal at the meetings and they wanted more such opportunities: "Would love to meet more people who are stakeholders in their learning."

The participants in this study expressed positive feedback about the opportunity to meet in a collegial setting with university faculty and board support staff to discuss common concerns and challenges. One participant stated: "Love the partnership and the professionalism." while another participant explained: "Working with peers (teachers, principal, board staff, and university faculty) has been an insightful experience. The outcome of being involved and learning from others has been beneficial to my teaching practice." Participants appreciated the professionalism and intellectual focus of the experience. Some participants liked getting to know their colleagues and also being supported in acknowledging that some strategies may not yet have worked: "Collaboration allowed us to discuss 'what worked' and the power of 'not yet' in order to improve our strategies."

\section{How does the collaborative inquiry process contribute to increased competencies and changes in pedagogical practices?}

The participants were grateful for learning new teaching skills and strategies. They were affirmed in their practice and had the chance to hear about new approaches from peers and fellow participants. One participant noted: "The discussion and sharing ideas were the most valuable piece for me. I feel more confident in my abilities to achieve the goals I have set for my students." For some participants, this was the first time that they had reflected on how their classroom teaching might affect outcomes of the provincial assessments of Grades 3 and 6. One teacher stated: "I've thought more about my practice than I ever had before. How do I improve scores?" Statements such as the following attest to the perceived value of involvement in the collaborative inquiry process: "The research initiative has allowed me to self-reflect on my own teaching practices and how to improve student learning," and "I am able to take time to critically reflect on my student learning and WHY they learn." Participants were grateful to have the time and impetus to focus on their professional learning and specific goals to improve student 
achievement. The classroom teachers tried many new strategies generated through group discussions to achieve the goals of the collaborative inquiry process.

\section{How did the collaborative inquiry initiative enhance student learning and skills?}

The changes in student learning and engagement related to persistence and task completion were demonstrated through teachers' reports on indicators of success outlined in their Research Action Plans. The participants had collected pedagogical documentation and data, such as work samples, student comments, time on task measures, photos, rubrics, and other artifacts. Teachers indicated that there were marked improvements in: students' ability to stay with a challenging task, quality and quantity of writing samples, completion of mathematics questions with supporting evidence, levels of self-regulation, focus during meditation activities, and positive attitudes towards difficult assignments. Some of the teachers' comments included: "Evidence was good...physically seeing the progress of the students," and "Awareness is the key and reflecting on my practice has contributed to making changes to my teaching strategies. I have also noticed my students have gained a greater awareness of the importance of independence, persistence, and grit."

The following examples describe indicators of success as reported by educators in the Research Action Plans according to the two main inquiry focus areas.

Inquiry Focus: Students improve perseverance and grit to complete tasks and increase stamina

\section{Indicators:}

- Homework completion improved overall;

- Less student reliance on teacher support;

- Greater number of students willing to share work;

- Increased time spent on independent work;

- Quantity and quality increased in choice assignments;

- More task completion in class;

- Increase of work focus from 8 minutes to 20 minutes; and

- Students created their work list every day, which resulted in less unfinished work/homework.

Inquiry Focus: Students demonstrate increased levels of accountability and independence in the learning process

\section{Indicators:}

- More ownership in reviewing work before handing it in;

- Increased pride in the draft process of writing and seeing it as a continuum of learning;

- Begin to use the newly introduced rubric for their other activities in class;

- Started showing initiative by requesting the teacher for conferencing;

- Students acknowledged times when they were stuck verbally using the phrase "My brain is on fire," with a positive attitude; 
- More persevering and engaging in difficult tasks without giving up;

- Demonstrated improvements in writing quality and writing productivity;

- Increased confidence in skills and abilities by striving for higher levels of achievement;

- After "Christian Meditation" sessions, the students showed increased levels of focus and acts of kindness;

- Increased levels of self-regulation and self-direction in task completion; and

- Reported ability to celebrate "not yet" as a means of bumping up their work.

These reports from teachers are clear indicators of improvements in the areas of persistence, focus, confidence, task completion, and self-direction. Overall, the classroom teachers noted increases in positive behaviours and less disruptive behaviours, indicative of students' greater self-esteem as learners. Students embraced the instructional strategies encouraging them to be independent and confident learners. The collaborative inquiry process with a focus on student engagement yielded positive effects on student learning.

\section{What are the enablers and challenges to developing collaborative inquiry teams in education?}

There were various enablers and challenges to the CITE model used for collaborative inquiry. The enablers included having resources such as the school principal, SWS teacher, the Literacy/Numeracy resource teacher, and faculty facilitators in the group. On participant noted, "Teachers were supported in their understanding of student learning...in developing a problem. Facilitators helped teachers focus their questions and their strategies". Other enabling conditions were the supply teacher coverage provided and the school-based site location. A teacher stated: "Being able to discuss strategies and next steps for our students in a collaborative setting is helpful." Participants seemed to enjoy the SURE videos as a catalyst for discussion and greatly appreciated the Research Action Plan templates to guide the collaborative inquiry process with ample time for discussion. A participant reported: "We learned how to utilize the skills and strategies developed through the Research Action Plan Cycle and effectively apply this process to our individual classroom focused goals." The use of the D2L site was useful, for most participants: "Finding out about D2L allowed me to think broader and how my classroom research could impact other classrooms within my school and across the Board. D2L allows teachers to share their reflective practices to a wider audience. The potential is limitless."

The challenges reported by participants include the short period of five months (some would have liked a year-long initiative): "Time...not enough time to gather data and share ideas," and "Would have been great to have a 10-month initiative...to gather more data." The need to "let go" of preconceived notions regarding student learning processes and teacher control was articulated as a challenge by one participant: "Overcoming some of my own challenges when identifying and implementing specific strategies to students, i.e.: letting go of some control." Some participants also expressed a desire to learn more about the potential usages of the D2L learning management system for the collaborative inquiry process: "A challenge is finding out more about D2L." This tool was both an enabler and a challenge as not all participants were comfortable with using D2L. Another challenge expressed was that a baseline was not available in some cases to specifically discern changes in student learning or task completion: "Need more depth and base... and that will come." 
Participants identified release time, school board personnel support, structure of CITE sessions and professional facilitation as enablers. Challenges included the limited opportunity for total immersion in the collaborative inquiry process over a five-month period and lack of familiarity with D2L. As expected, more time and better use of the learning management system were noted as potential conditions to further enable the collaborative inquiry and research.

\section{Discussion}

The present collaborative inquiry initiative is an innovative educational research community model because it has demonstrated positive effects for both the participants and the students in their school, utilizing human resources and the technological support of a learning management system. Similarly to Martinovic et al. (2012), the present study affirmed that focused attention, support from researchers and administrators, and structured activities foster teacher research acumen and are appreciated by teachers. The study also showed that collaborative inquiry school-based teams can become a catalyst for educational research, $\mathrm{KMb}$, and student learning (Edelstein, 2015). The following table summarizes the two main themes that emerged from the findings of the present study are Teachers as Engaged Researchers and Students as Engaged Learners (Table 1)

Table 1

Teachers as Engaged Researchers and Students as Engaged Learners

Theme

Teachers as Engaged Researchers

Students as Engaged Learners
Subtheme
Professional Growth and Re
School Culture of Inquiry

Enablers for Collaborative Inquiry

Teacher Pedagogical Practices

Classroom Culture of Inquiry

Enablers for Student Learning

The first main theme "Teachers as Engaged Researchers" included the subthemes of professional growth and resources, school culture of inquiry, and enablers for collaborative inquiry. The concept of "teachers as engaged researchers" was evident throughout the participants' comments affirming their professional growth through the CITE initiative. Participants were initially wondering about the focus of the study, but quickly embraced the excitement of documenting their own learning and that of their students. The school culture became one of inquiry, questioning, and reflection as noted in the study by Kyei-Blankson (2014). As was found by Cooper (2011), the KMb efforts that included teachers and researchers in the same sessions created a culture of inquiry, not only in the CITE initiative but also throughout participants' 
classrooms and the school. An interesting finding was that the use of the D2L learning management system site was deemed as both an enabler and a challenge. It was useful to have all the materials posted on one site and to have a forum for discussion, but using this strategy without training presented challenges. Perhaps the use of an online platform for collaborative inquiry, as also suggested by Cooper (2014), is an area for future study. Other enabling conditions were the professional nature of the sessions, with the provision of agendas, minutes, presentations, and templates for structured activities. There was also ample time for discussion and questioning in a stimulating setting with collegial support. The SURE videos, addressing a variety of topics related to educational research, were of interest to the participants, especially because the videos included familiar school board staff and local schools. The videos stimulated discussion and affirmed participants' perspectives on the importance of collaborative inquiry in the process of building common understandings across educational settings. The trust and empathy that is needed for a successful collaborative inquiry were formed through the communication, commitment, and continuity of the research team (Ponte, et al., 2004). The fact that the university faculty members had previously been members of the school board may have nurtured a sense of trust in the group and facilitated the mobilization of knowledge from theory to practice.

The second theme, "Students as Engaged Learners," emerged as CITE participants became more aware of pedagogical issues in their learning environment and learned how to seek solutions for student challenges. The participants increased their familiarity with the research process and collaborative inquiry teams. Focusing on the development of teacher as researcher identities and questions of inquiry related to student engagement and learning, participants created a culture of inquiry in their classrooms. Teachers reported that students wanted to know more about their teachers' research and the teachers strived to complete exemplary work since it was being examined as pedagogical documentation. Students became excited about the research and the teachers' use of such strategies as writing guidelines, timed tasks, and meditation to promote persistence, resilience, task completion, and community building. The teacher participants reported that students were able to sustain greater attention to tasks and were more independent and resourceful during their self-directed work periods. The results of the present collaborative inquiry were encouraging, and there is the possibility that students might also become researchers in exploring their areas or questions of inquiry.

The findings of this study showed that the CITE model of collaborative inquiry-a research partnership between teachers, school board administrators, and university faculty facilitators-is an effective vehicle for addressing school improvement plans and increased student learning. Such collaborative inquiry promotes knowledge creation and mobilization, ultimately enhancing student engagement and learning.

The following three simple formulas reflect the main themes identified:

$\begin{array}{lllll}\text { Teachers } & + & \text { Collaborative Inquiry } & = & \text { Teacher Researchers } \\ \text { Teacher Researchers } & + & \text { Knowledge Mobilization } & = & \text { Student Engagement } \\ \text { Student Engagement } & + & \text { Evidence-based Strategies } & = & \text { Student Learning }\end{array}$

There are various factors in the CITE initiative that could be considered as limitations to this study. The participants volunteered to be involved in this collaborative inquiry, which may have contributed to the positive attitudes towards research. Additionally, the presence of the principal may have been deemed to keep teachers engaged in the process and affect the outcome of the 
collaborative inquiry as teachers may have verbally stated what they may have perceived the principal would find appropriate (written comments were confidential). It is well documented that principals play a pivotal role in school based collaborative inquiry processes (Fullan \& Langworthy, 2014). It would be interesting to replicate this study with teachers and principals of differing schools and over a longer period to allow for the inquiry cycle to be completed over one school year.

\section{Conclusion}

From the present study, it is evident that there are many positive results and outcomes to the collaborative inquiry initiative, formed in a partnership between a school board and university faculty members. The positive effects include teachers' increased familiarity with research and pedagogical practices to enhance student engagement in learning. It can be deduced that the collaborative inquiry process was also an excellent vehicle to utilize $\mathrm{KMb}$ resources such as the SURE videos to address the challenges to teachers' utilisation of educational research as outlined by Martinovic et al. (2012). The use of videos, structured sessions, faculty and technological support, and a school improvement focus created the compelling purpose of CITE and ensured its sustainability over five months and into the future. Participants expressed ongoing appreciation for the learning experience stating that "it brought to life the school improvement plan" and created a "culture of responsive teaching." The CITE sessions were facilitated through a board budget, in collaboration with the SWS teacher and the school principal. Perhaps, with limited resources, these sessions could be supported by planning time periods renamed as research time periods, with focused usage of at least one monthly period of this research time devoted to a collaborative inquiry initiative. It was affirming to note that participants embraced the opportunity to learn from the SURE videos, complete the components of the Research Action Plan, experiment with the D2L learning management platform, present their preliminary findings at a related conference, and embark on a writing project. Collectively and collaboratively, this CITE initiative named, framed, and proclaimed authentic and applied educational research, utilizing $\mathrm{KMb}$ strategies and resulting in enhanced teacher and student engagement and learning. 


\section{Acknowledgements}

Thanks are extended to Asha Mistry and Jessica Perron, students at the Nipissing University, Schulich School of Education, for their assistance. The Waterloo Catholic District School Board provided invaluable support to the CITE Initiative. Many thanks are extended to all CITE participants for their enthusiastic involvement. 


\section{References}

Blakemore, H. (2012). Emergent teacher-researchers: A reflection on the challenges faced when conducting research in the English classroom. English Teaching: Practice \& Critique, 11(2), 59-69.

Charmaz, K. (2006). Constructing grounded theory: A practical guide through qualitative analysis. Thousand Oaks, CA: SAGE Publications.

Cooper, A. (2011). Knowledge mobilization intermediaries in education across Canada Unpublished Doctoral Dissertation. University of Toronto, Toronto, Ontario.

Cooper, A. (2014). The use of online strategies and social media for research dissemination in education. Education Policy Analysis Archives, 22(88/89), 2-27

Creswell, J. W. (2009). Research design: Qualitative, quantitative, and mixed methods approaches. Los Angeles, CA: Sage.

Fullan, M., \& Langworthy, A. (2014). Rich seam: How new pedagogies find deep learning. London: Pearson

Gabriel, M., Doiron, R., Baldacchino, A., McKenna, J., \& O’Keefe, A. R. (2012). Play in early learning programs: Beliefs, practices, and professional development. Final Research Project prepared for Early Childhood Development Association. Charlottetown, PEI.

Gore, J. M., \& Gitlin, A. D. (2004). [Re]Visioning the academic-teacher divide: Power and knowledge in the educational community. Teachers \& Teaching, 10(1), 35-58.

Grierson, A., Tessaro, M. L., Grant, C., Cantalini-Williams, M., Denton, R., Quigg, K., \& Bumstead, J. (2012). The bricks and mortar of our foundation for faculty development: Book study within a self-study professional learning community. Studying Teacher Education, 8(1), 87-104.

Guest, G., MacQueen, K., \& Namey, E. E. (2012). Applied thematic analysis. Thousand Oaks, CA: Sage.

Hannay, L., Wideman, R., \&Seller, W. (2010). Professional learning to reshape teaching. Toronto, ON: Elementary Teachers Federation of Ontario.

Hine, G. C., \& Lavery, S. D. (2014). Action research: Informing professional practice within schools. Issues in Educational Research, 24(2), 162-173.

Kane, R. G., \& Chimwayange, C. (2014). Teacher action research and student voice: Making sense of learning in secondary school. Action Research, 12(1), 52-77.

Kitchen, J., Ciuffetelli Parker, D., \& Gallagher, T. (2008). Authentic conversation as faculty development: Establishing a self-study group in a faculty of education. Studying Teacher Education, 4(2), 157-171.

Kuntz, A. M., Presnall, M. M., Priola, M., Tilford, A., \& Ward, R. (2013). Creative pedagogies and collaboration: An action research initiative. Educational Action Research, 21(1), 4258. 
Kyei-Blankson, L. (2014). Training math and science teacher-researchers in a collaborative research environment: Implications for math and science education. International Journal of Science \& Mathematics Education, 12(5), 1047-1065.

Latta, M. M., \& Buck, G. (2007). Professional development risks and opportunities embodied within self-study. Studying Teacher Education, 3(2), 189-205.

Leigh, J. (2016). An embodied perspective on judgements of written reflective practice for professional development in higher education. Reflective Practice, 17(1), 72-85.

Martinovic, D., Wiebe, N., Ratković, S., Willard-Holt, C., Spencer, T., \& Cantalini-Williams, M. (2012). "Doing research was inspiring": Building a research community with teachers. Educational Action Research, 20(3), 385-406.

McIntyre, D. (2005). Bridging the gap between research and practice. Cambridge Journal of Education, 35(3), 357-382.

Miles, M., \& Huberman, M. (1994). Qualitative data analysis: An expanded source book (2 $2^{\text {nd }}$ ed.). Thousand Oaks, CA: Sage.

Ontario Ministry of Education. (2016). Capacity building series. Inspire. [Document series]. Retrieved from: http://www.edu.gov.on.ca/eng/literacynumeracy/inspire/research/capacityBuilding.html

Ponte, P., Beijard, D., \& Ax, J. (2004). Don't wait till the cows come home: Action research and initial teacher education in three different countries. Teachers \& Teaching, 10(6), 591 621.

The SURE Network. (2014). Research to practice. [Video series]. Retrieved from: http://www.surenetwork.ca/category/videos/

Williams, D., \& Coles, L. (2007). Teachers' approaches to finding and using research evidence: An information literacy perspective. Educational Research, 49(2), 185-206. 